\title{
Object-based selection within and beyond the focus of spatial attention
}

\author{
MARK B. LAW and RICHARD A. ABRAMS \\ Washington University, St. Louis, Missouri
}

\begin{abstract}
In a series of experiments, we examined the effect of perceptual objects on visual attentional processing in the presence of spatially cued attentional selection. Subjects made speeded judgments about two visual elements that were either both on the same object or on two different objects. Judgments were faster when the elements were on the same object than when they were on different objects, revealing an object advantage. Importantly, the object advantage remained even when either exogenous or endogenous spatial cues were used to direct the subjects' attention to a part of the display, contrary to earlier findings of other researchers. The object advantage, however, did disappear when the stimulus duration was shortened substantially. The results show that object-based selection is pervasive and is not diminished by the act of selectiveattention. The results are discussed in terms of their implications for the mechanisms that underlie attentional selection.
\end{abstract}

In order to make sense of a complex visual world, humans possess attentional mechanisms that permit the selection of a portion of a scene for detailed processing. The selection is determined in part by events that occur in the scene, such as a flashing light or a waving hand, and in part by goals of the observer, such as the desire to find one's friend in a crowd. In addition to these stimulus-driven and goal-directed attentional mechanisms, respectively, it is becoming increasingly clear that the objects that are present in a scene also play an important role in the guidance of selective attention. Such a role is sometimes referred to as object-basedorienting of attention. In what follows, we will briefly review the evidence for object-based orienting, and we will examine a potential boundary condition for the phenomenon: the extent to which object-based orienting can occur outside the focus of attention.

\section{Object-Based Attention}

Object-based attentional selection can be thought of as a hybrid mechanism that combines aspects of both goaldirected and stimulus-driven selection. For example, Duncan (1984) had subjects view a display containing two superimposed objects. He found that the subjects were more accurate in reporting two features from one object than they were in reporting one feature on each of the two objects. Thus, although top-down instructions determined the object that was to be attended (as in goal-directed selection), the stimulus configuration determined the ease

Portions of the research reported in this article were submitted in partial fulfillment of the requirements for a doctoral degree by M.B.L. The research reported here was supported by Grants MH45145 from the National Institutes of Health and BCS-0079594 from the National Science Foundation.Correspondence concerning this article should be addressed to R. A. Abrams, Department of Psychology, Washington University, St. Louis, MO 63130 (e-mail: rabrams@artsci.wustl.edu). with which the selection could be made (as in stimulusdriven selection).

A study by Egly, Driver, and Rafal (1994) also demonstrated a way in which stimulus-driven attention may be affected by objects. Egly et al. had subjects attend to one end of one of two rectangles in a display by flashing a light at the end of the rectangle. The flashing light was presumed to have engaged stimulus-driven attentional mechanisms. The subjects were then asked to detect the onset of a target that appeared at the cued location, in the opposite (i.e., uncued) end of the cued object, or in the other, uncued object. The important result was that there was an object advantage: Subjects were faster to detect targets in the uncued end of the cued object, as compared with those in the uncued object. This was true even though those two locations were equidistant from the central fixation marker and from the location that had been cued. Thus, the objects in a scene play an important role in the guidance of selective attention - even concurrently with ongoing stimulusdriven attentional selection. (Abrams \& Law, 2000, have recently shown that Egly et al.'s results also hold for endogenously cued, goal-directed attention.)

A number of other results also have corroborated the importance of objects in selection from scenes. Yantis and Hillstrom (1994) showed that new objects can capture attention when they initially appear, even if the appearance does not involve a change in luminance in the scene (see also Oonk \& Abrams, 1998; but see Gellatly, Cole, \& Blurton, 1999). Tipper, Driver, and Weaver (1991) showed that inhibition of return, an inhibitory attentional phenomenon, applies to objects in a scene and will move with an affected object if the object moves (see also Abrams \& Dobkin, 1994; Abrams \& Pratt, 2000; Christ, McCrae, \& Abrams, 2002). Kahneman, Treisman, and Gibbs (1992) reported a similar object benefit for identification of letters in boxes that moved. Finally, Baylis and Driver (1993) showed that 
perceptual set can determine whether a display is seen as one or two objects and that the perception, in turn, determines the ease with which subjects can make comparisons between elements in the scene. These results, when taken together, establish an important role for objects in scenes. It is not simply the case that attention operates upon an unstructured representation of the world. Instead, attentional selection appears to be accomplished by access to a representation that contains in it information about the objects that are present in a scene.

\section{Limitations of Object-Based Attention}

Lavie and Driver (1996) used a variation of Duncan's (1984) paradigm to examine potential limitations of objectbased attention. They had subjects view two intersecting dashed lines to compare the lengths of two target elements that differed from the others in color (the targets were either a dot or a dash in a line). When the target elements were both on the same line, subjects made the comparison more quickly than when each element was on a different line-revealing the typical object advantage, such as that shown by Duncan.

Lavie and Driver (1996) investigated whether stimulusdriven attention might modulate the object advantage that they reported. In one experiment, they cued attention to one side of the display (via a transient visual event in the periphery) prior to presentation of the stimuli on each trial. Subjects were faster, not surprisingly, when the target elements were both on the side that had been cued. Most important, the object advantage that had been observed previously was no longer present. Lavie and Driver concluded that the object advantage does not occur when spatial attention is narrowly focused (Dagenbach, Goolsby, Neely, \& Dudziak, 1997, have also reported results consistent with this conclusion). In particular, the object advantage did not appear to extend beyond the focus of attention.

Lavie and Driver's (1996) conclusion represents a significant challenge for our understanding of the attentional system. Their results imply that objects may have a limited role, with the object advantage being reduced or eliminated for portions of a scene that are outside the focus of attention. Indeed, it would seem that there might be some utility to a mechanism that, when attention is narrowly focused, may permit (or require) an observer to disengage some automatic attentional mechanisms (such as, in this case, the selection of objects) in favor of the endogenous selection that has been made. Such a mechanism is not without some precedent in the attention literature. For example, Folk, Remington, and Johnston (1992; see also Folk \& Remington, 1999) have shown that the capture of attention by some feature of a display (e.g., color) may depend on attentional control settings induced by the instructional demands of the experimental task (such as whether the target is defined by its color or by some other attribute). Similarly, Yantis and Jonides (1990) have shown that endogenous orienting can sometimes override the capture of attention by abrupt onsets. In an analogous way, ob- jects in a scene may affect selection only in the absence of some specific top-down instruction guiding selection.

Nevertheless, Lavie and Driver's (1996) conclusion also appears to be somewhat at odds with the work by Egly et al. (1994) noted earlier, as well as with similar experiments by Abrams and Law (2000). These researchers showed that attention can be directed to part of an object, yielding an attentional benefit for detection or discrimination of targets in that place, while at the same time uncued parts of the cued object can enjoy an advantage over other objects that had not been cued at all. In other words, object-based selection appeared to operate simultaneously with spatially cued selection.

Furthermore, a recent study by Lamy (2000) has failed to replicate Lavie and Driver's (1996) results under conditions very similar to those examined in the original Lavie and Driver study. Unfortunately, no explanation was offered for the discrepant results. Thus, our goal was to reexamine the issue in an attempt to adjudicate between the two opposing views. As may be clear from the preceding discussion, resolving the discrepancy could have important implications for models of the attentional system.

\section{Overview of Experiments}

In what follows, we reexamined the extent to which object-based and space-based orienting may be mutually exclusive. We studied questions about the nature of the stimuli, the types of spatial cues, and aspects of stimulus timing and probability. In Experiment 1, we developed a new type of stimulus in order to examine the issues under consideration. The stimulus had an advantage over those used previously by others, in that the features to which the subjects attended were intrinsic parts of the objects in the display. Experiment 1 established that these new stimuli were capable of yielding an object advantage. In Experiment 2, we examined the extent to which the object advantage occurred in the presence of spatial cues directing attention to only a subset of the display. Exogenous cues were used in Experiment 2. The same question was addressed in Experiment 3 using endogenous cues. In Experiment 4, we attempted to more closely replicate the cue used by Lavie and Driver (1996). In each of Experiments 2-4, in which cuing was used, we detected an object-based component simultaneously with a cuing benefit (and extending beyond the area cued), contrary to the findings of Lavie and Driver but consistent with the report by Lamy (2000). Finally, in Experiment 5, we reduced the duration of stimulus exposure to match that used by Lavie and Driver in their experiment in which no object advantage was detected. Under those conditions, the object advantage disappeared, suggesting that exposure duration plays an important role in the phenomenon and resolving some of the discrepancies in the literature.

\section{EXPERIMENT 1}

In this experiment, we attempted to replicate the object advantage reported by Lavie and Driver (1996) in a situa- 


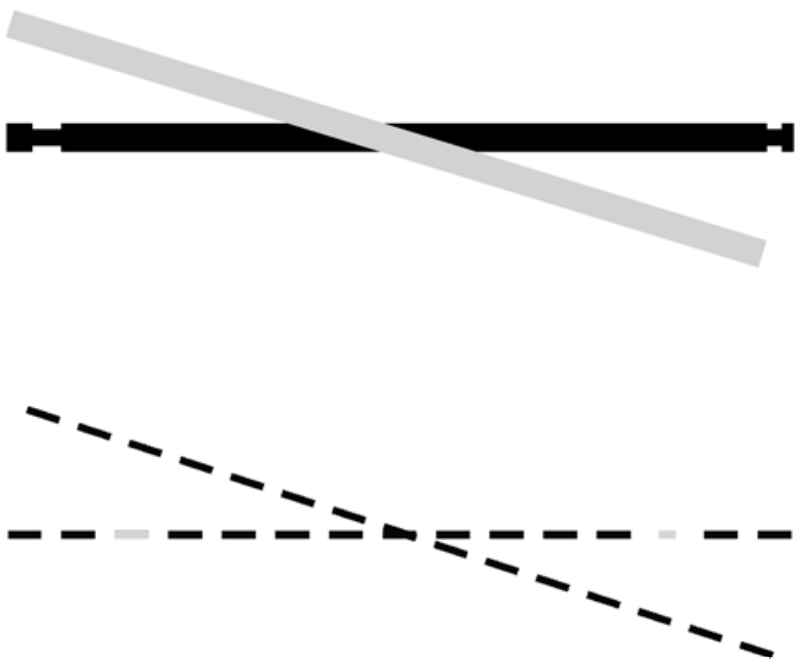

Figure 1. Top: example of the stimuli used in the present experiments. The subject's task was to compare the size of the two notches (same or different). Bottom: stimuli used by Lavie and Driver (1996), in which the subject's task was to compare the lengths of the two odd-colored line elements.

tion without cued attention, but in which new stimuli were used. We sought to develop new stimuli for further investigation of the object advantage because we wanted to be certain that the attributes that our subjects were asked to judge would be regarded as intrinsic parts of the objects in the display and would not be seen as objects in their own right. In this way, the to-be-judged elements would be unlikely to be selected themselves, and any effects obtained would be attributable to a true object advantage in selection, whenever such effects were observed. As can be seen in the top panel of Figure 1, our subjects were asked to compare the sizes of two notches that were removed from one or two rectangular bars present in the display. Sometimes the notches were equal in size, and sometimes they were unequal. These objects contrast with those used by Lavie and Driver and Lamy (2000). Those researchers used dashed lines, as in the bottom panel of Figure 1. The subjects' task in the earlier study was to compare the lengths of the two odd-colored elements (they could be either dots or dashes, or one of each). Arguably, the dashes themselves might be regarded as objects in their own rightpossibly complicating any conclusions made about selection in general on the basis of their results.

\section{Method}

Subjects. In return for class credit, 17 undergraduates participated in a single 1-h session. They all reported having normal or corrected-to-normal vision and were naive as to the purposes of the experiment.

Stimuli. The stimulus display was composed of two intersecting solid bars centered on the computer screen. Each bar subtended $11.25^{\circ}$ of visual angle and was $0.45^{\circ}$ thick; one bar was always horizontal, and the other bar was always rotated $17^{\circ}$ clockwise from the horizontal. One bar in each display was red, and the other was blue. Bar color assignment was randomly determined for each trial. Since the bars crossed in the middle, the bar that appeared in the fore- ground was randomly determined for each trial. As can be seen in Figure 1, the bars were sometimes notched. The notches were formed by creating either a $0.4^{\circ}$ long or a $0.2^{\circ}$ long constriction with a thickness of $0.24^{\circ}$.

Each display had two target notches, either one on each bar or both on opposite ends of the same bar. The center of each notch was either $1.25^{\circ}$ or $1.95^{\circ}$ from the nearest end of the bar; this eccentricity was randomly determined for each trial. The notches were the same size on half of the trials and different sizes on the other half. There were as many trials with two small notches as with two large notches. When the notches were different sizes, the notch size assigned to a given bar end was randomly determined. The notches appeared on each side of the display equally often; they also appeared on the horizontal bar as often as they appeared on the diagonal bar.

Procedure. Testing was conducted in a dimly lit sound-attenuated room. The subjects were seated in front of a computer monitor, and their heads were steadied with a chinrest. At the beginning of each trial, a fixation cross was shown at the center of the screen for $1,000 \mathrm{msec}$. The subjects were told to fixate upon the cross. The cross was then removed, and the bar stimuli were presented. The bars remained visible for $186 \mathrm{msec}$, after which a blank screen was shown. The subjects' task was to judge the relative size of the two notches that were present. The subjects were to press, as quickly as possible, the " $\mathrm{z}$ " key if the notches were the same size or the "/" key if they were different sizes. The next trial began $250 \mathrm{msec}$ after the subject responded on correct trials. If the response was incorrect, an error message was presented for $1,500 \mathrm{msec}$, followed by a 250 msec delay. If the subjects misjudged the stimuli, they were also reminded of the key-response assignment; messages were also presented if the response was too early (100 msec or less), or too late (1,000 msec or longer). The subjects were given performance feedback and an opportunity to take a short break every 40 trials.

Design. A block of 20 practice trials was presented at the beginning of each session. These trials were chosen at random from the full set of trials. Practice was followed by 10 experimental blocks of 80 trials each. The proportions of trials in each condition were based on the proportions successfully used by Lavie and Driver (1996). Of the 80 trials in a block, 64 were in the near condition. On those trials, the two notches to be judged were each on the same side of the display and on different bar objects. The remaining 16 trials were split between the far condition, in which the notches were on different bars and different sides of the display, and the object condition, in which the notches were both on the same bar and on different sides of the display. An example of the object condition is shown in the top panel of Figure 1.

\section{Results}

Mean reaction times for correct trials in the near, far, and object conditions are shown in Figure 2. The subjects were quickest to judge notch size in the object condition (646 msec), next quickest in the near condition $(653 \mathrm{msec})$, and slowest in the far condition $[661 \mathrm{msec} ; F(2,32)=4.3$, $p<.05]$. Comparing the far condition with the object condition shows that the object advantage was $15.0 \mathrm{msec}$ $[t(16)=2.6, p<.05]$. That is, the subjects judged the notches quicker when they were both on the same bar than when they were on different bars but a similar distance from each other. This clearly shows that this method and these stimuli are capable of detecting an object advantage. The subjects made a premature response (latency less than $100 \mathrm{msec}$ ) or a late response (latency greater than $1,000 \mathrm{msec}$ ) on fewer than $1 \%$ of the trials. The overall response accuracy was $89 \%$; there was no difference in accuracy between conditions $[F(2,32)<1]$. 


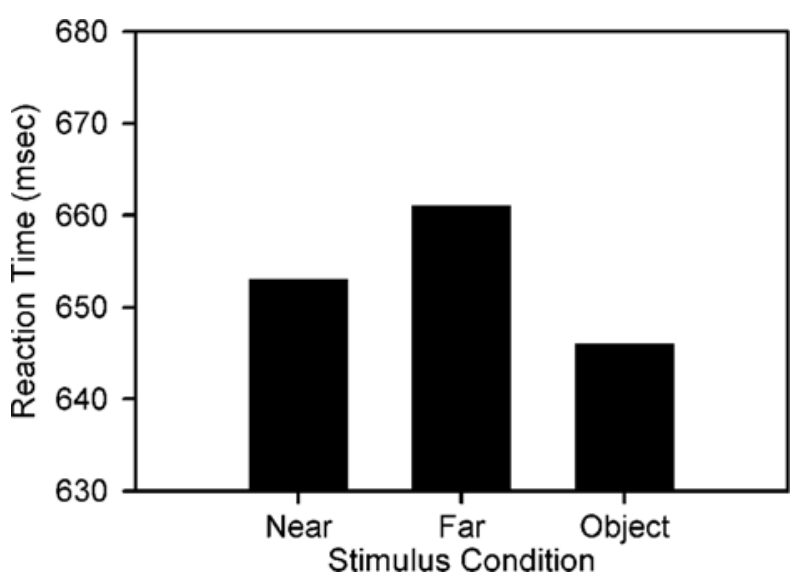

Figure 2. Results from Experiment 1. Here, an object advantage is revealed by the faster response times in the object condition than in the far condition.

\section{Discussion}

The most important outcome of this experiment was the clear confirmation that the new stimuli we developed and the presentation times used were capable of producing an object advantage. Rather than form the stimuli from a collection of grouped line segments, as Lavie and Driver (1996) and Lamy (2000) had done, the stimuli we used were solid bars. The judged attributes (notches) required the processing of the objects (the bars), so it is unlikely that the notches could have been perceived as separate objects. The 15-msec object advantage found was similar in size to the 16-msec advantage found by Lavie and Driver (1996, Experiment 3, the experiment most comparable to the present one). The experiments that follow built upon this foundation.

\section{EXPERIMENT 2}

Lavie and Driver (1996) claimed that spatial attention eliminates object-based attention. That is an interesting claim because it places a new limitation on the extent of object-based attention. However, that result does not seem consistent with some earlier findings, such as those of Egly et al. (1994) and Abrams and Law (2000), who showed both spatial cuing and an object advantage. It could be that the cue Lavie and Driver used created some sort of concurrent, whole-object cuing that obliterated object-based selection in their paradigm.

Lavie and Driver (1996) cued attention to one side of the display by presenting the ends of the dashed-line objects on the cued side of the display before presenting the rest of the lines. However, it has been established that when attention is cued to a part of an object, attention then radiates throughout the entire object and parts of the object that were not directly cued may benefit (Abrams \& Law, 2000; Egly et al., 1994; Macquistan, 1997; Vecera, 1994). Thus, the cues used by Lavie and Driver might have caused attention to select both line objects, and not just the spatial region near the cue. For instance, Abrams and Law (2000) successfully cued attention to a whole object by presenting a peripheral cue in the area of a blank display that would later be occupied by one end of an object. Subjects then made facilitated judgments of a discrimination target when it was located on the uncued end of an object that was spatially coincident with the cue. If Lavie and Driver's cue operated in the same way, it might have been unlikely for their cuing experiment to detect an object advantage, since both objects would have been selected, consistent with their failure to find an object advantage in the presence of cuing. However, it should still be possible for them to detect a spatial cuing effect, since the predictive peripheral cue would still be expected to speed judgments of targets on the cued end of the object, as compared with the uncued end (Abrams \& Law, 2000; Egly et al., 1994). Therefore, it is possible that the pattern of results Lavie and Driver interpreted as evidence of the modulation of object-based attention by spatial attention was an artifact of the cue they used, because that cue was both an effective spatial cue and an effective object cue.

The present experiment avoided that criticism by using a spatial cue that never occupied the same locations as the bar objects. The cue consisted of three white dots that appeared on the side of the display where the notches were most likely to appear. This three-dot cue directed attention to a broader region of space and not directly to the bars and, therefore, seems less apt to have caused selection of the objects.

\section{Method}

Subjects. In return for class credit, 20 undergraduates participated in a single 1-h session. They all had normal or corrected-to-normal vision, were naive as to the purposes of the experiment, and had not participated in Experiment 1.

Procedure and Stimuli. The sequence of events on a trial is shown in Figure 3. All aspects of the experiment were the same as those in Experiment 1, except for the differences described here. As was noted earlier, the subjects were cued to attend to one side of the display by an array of three dots presented beside the bar objects on the cued side. Also, the bar display was rotated so that the bars were each at an equal and opposite angle from the horizontal, to ensure that the bar ends were centered in the cued area. Seventy percent of the trials were validly cued near condition trials in which the two notches to be judged were both on the cued side of the display and on different bars. (Note that near refers to the distance between the two notches, not that between the cue and the notches.) The remaining trials were evenly divided between the nonvalid near condition, in which the notches were on different lines on the side of the display opposite the cue, the far condition, in which the notches were on different lines and different sides of the display, and the object condition, in which the notches were both on the same bar and on different sides of the display. As is shown in Figure 3, the space near the bars was cued by presenting three white dots, each $0.5^{\circ}$ in diameter, on the cued side of the display $71 \mathrm{msec}$ prior to presenting the bar objects. Note that the dots were placed in locations that would be near but not overlapping with the bar ends. The subjects were instructed that the cue would usually indicate the side of the display on which the notches would both appear. They were also encouraged not to move their eyes from the center of the screen.

Design. A block of 20 practice trials was presented at the beginning of each session. These trials were chosen at random from the 


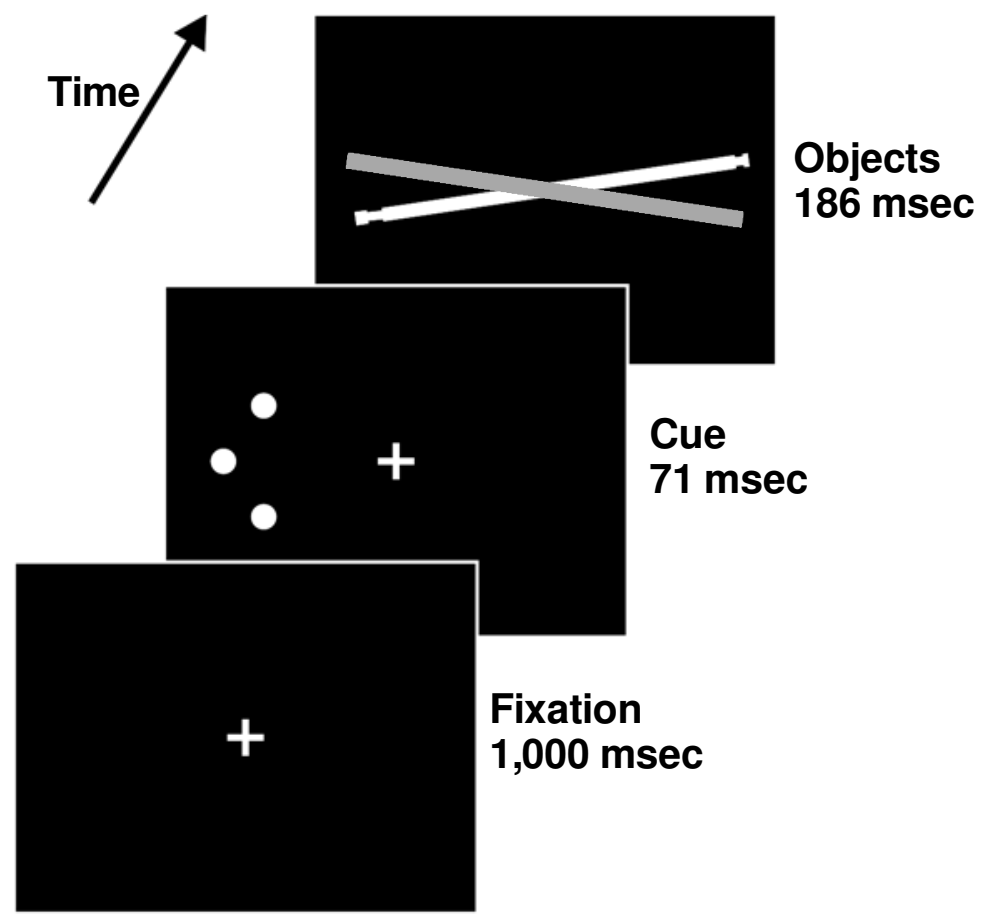

Figure 3. Events on a trial of Experiment 2. The three dots that formed the cue were displayed in the spaces around and between the bar objects. The example shown in the figure is from the object condition, in which the two notches are on the same object.

full set of trials. Practice was followed by 10 experimental blocks of 80 trials each. Of these 80 trials, 56 were valid near condition trials. The remaining 24 trials were evenly divided among the nonvalid near, the far, and the object conditions. The cue accurately predicted the side of the display on which both notches appeared on $70 \%$ of the trials.

\section{Results}

Mean reaction times for the valid near, nonvalid near, far, and object conditions are shown in Figure 4. The subjects were quickest to judge notch size in the valid near condition (643 msec), next quickest in the nonvalid near condition (649 msec), next quickest in the object condition $(651 \mathrm{msec})$, and slowest in the far condition [662 msec; $F(3,60)=6.3, p<.01]$. Comparing the far condition with the object condition shows that the object advantage was $11 \mathrm{msec}$ - that is, the subjects were quicker to make their judgment in the object condition, in which the notches were both on the same bar, than in the far condition, in which the notches were on different bars but at a distance from each other similar to that in the object condition $[t(20)=2.156, p<.05]$. There was also a spatial cuing effect of $6 \mathrm{msec}$ shown by the comparison of the valid near and the nonvalid near conditions $[t(20)=2.852, p<.05]$.

Finally, there was no difference between the nonvalid near and object conditions $[t(20)=0.356, p>.05]$. Given the clear object advantage, it might seem surprising that performance was as good in the nonvalid near condition. However, it is possible that the distance between the ele- ments being judged may have played a role in the present task-with responses generally quicker when the two elements being compared were closer to each otherresulting in a relative advantage for the nonvalid near condition. It is also possible that the speed in the nonvalid near condition was due to forward masking (Breitmeyer \& Ganz, 1976) of one or both of the notches in all the conditions except nonvalid near. In particular, the peripheral cue appeared briefly and immediately before presentation of the bar objects and in the vicinity of one or both of the notches in all the conditions except the nonvalid near condition. In that condition, both notches were on the uncued side of the display and, thus, could not be masked by the cue. Thus, it seems possible that latencies in the nonvalid near condition were unusually fast, as compared with all the other conditions, owing to the absence of masking there. That could explain the equivalent latencies in the nonvalid near and the object conditions but, of course, does not compromise the main question of interest regarding object effects and cuing.

The subjects made a premature response (latency less than $100 \mathrm{msec}$ ) or a late response (latency greater than $1,000 \mathrm{msec}$ ) on fewer than $1 \%$ of the trials. The overall response accuracy was $87 \%$. Overall errors differed somewhat across conditions $[F(3,60)=3.447, p<.05]$. There were fewer errors made in the valid near condition $(13 \%$ error rate) than in the nonvalid near condition [ $15 \%$ error rate; $t(20)=3.0, p<.01]$, providing further evidence of 


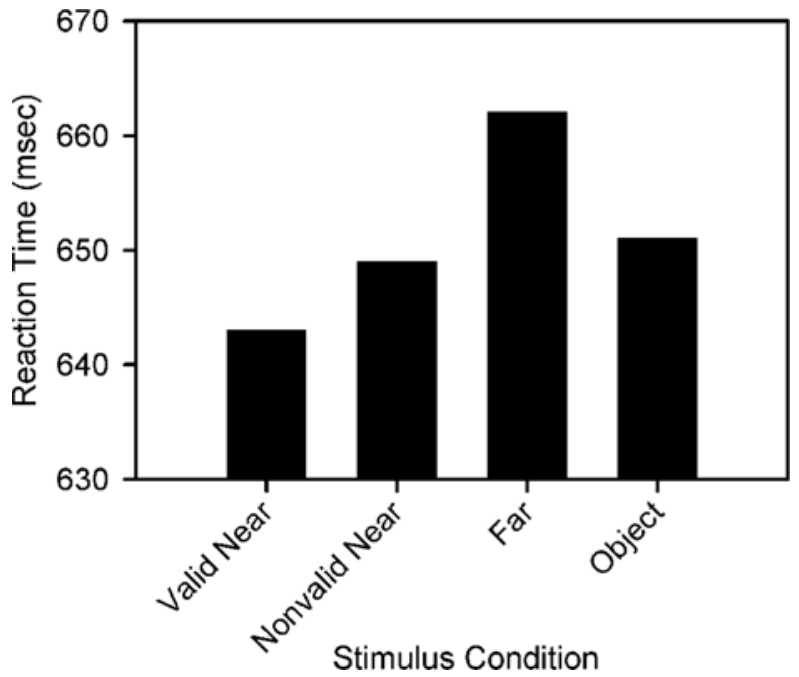

Figure 4. Results from Experiment 2. The difference between the valid near and the nonvalid near conditions indicates a spatial cuing effect, and the difference between the object and the far conditions indicates an object advantage.

a spatial cuing effect, but equivalent errors were made in the object condition ( $10.4 \%$ error rate) as compared with the far condition [9.5\% error rate; $t(20)=1.32, p>.05$ ]. Importantly, the pattern of errors that was observed did not support any speed-accuracy tradeoffs, since the fastest condition did not necessarily yield the largest number of errors.

\section{Discussion}

The important outcome of the present experiment is the observation of both spatial selective attention and an object advantage at the same time. This result casts doubt on the claim that focused spatial attention can eliminate objectbased attention. Because the main difference between the present experiment and Lavie and Driver's (1996) was the cuing technique used, it seems possible that the elimination of object-based selection by spatial attention that they found might have been caused by their particular method of cuing. However, alternatives are considered in what follows.

\section{EXPERIMENT 3}

Experiment 2 showed that spatial attention and objectbased attention could coexist when attention was exogenously cued. Attention can also be cued by means other than an exogenous cue. In particular, an endogenous cue can be used to direct attention to spatial locations (Posner, 1980) and to objects (Abrams \& Law, 2000). There is evidence to suggest that different mechanisms underlie exogenous and endogenous orienting. For instance, the time course is different for the development of the effects of exogenous and endogenous orienting (Jonides, 1981; Muller \& Rabbitt, 1989), the effects of these two types of orienting combine in an additive way (Riggio \& Kirsner, 1997), and under certain conditions endogenous cues can over- ride exogenous ones (Muller \& Rabbitt, 1989; Yantis \& Jonides, 1990). Also, inhibition of return, the tendency to orient attention more slowly to a recently attended location or object than to a new location or object, follows exogenous, but not endogenous, cues (Posner \& Cohen, 1984; Rafal, Calabresi, Brennan, \& Sciolto, 1989). Since there are many differences between these two types of cues, it cannot be taken for granted that the same phenomena will follow each cue. Thus, in the present experiment, we sought to discover whether endogenously cued spatial attention can modulate object-based attention.

\section{Method}

Subjects. In return for class credit 20 undergraduates participated in a single 1-h session. They all had normal or corrected-to-normal vision, were naive as to the purposes of the experiment, and had not participated in Experiments 1 or 2.

Procedure and Stimuli. This experiment was identical to Experiment 2, except that here the cue was a white arrow presented in the center of the display, at fixation, that pointed to the side where the notches were most likely to appear, as is shown in Figure 5. The size of the arrow was such that it would fit inside a $1.1^{\circ} \times 1.0^{\circ}$ box.

\section{Results}

Mean reaction times for the valid near, nonvalid near, far, and object conditions are shown in Figure 6. The subjects were quickest to judge notch size in the valid near condition $(639 \mathrm{msec})$, next quickest in the object condition $(665 \mathrm{msec})$, next quickest in the nonvalid near condition $(674 \mathrm{msec})$, and slowest in the far condition [678 msec; $F(3,63)=20.8, p<.001]$. Comparing the far condition with the object condition shows that the object advantage was $13 \mathrm{msec}$ - that is, subjects were quicker to make their judgment in the object condition, when the notches were both on the same bar, than in the far condition, when the notches were on different bars but a similar distance from each other, as in the object condition $[t(21)=3.242$, $p<.005]$. There was also a spatial cuing effect of $35 \mathrm{msec}$ shown by the comparison of the valid near and nonvalid near conditions $[t(21)=5.630, p<.001]$. Note that unlike Experiment 2, here reaction times in the object condition were faster than those in the nonvalid near condition $[t(21)=1.849, p=.08]$.

The subjects made a premature response (latency less than $100 \mathrm{msec}$ ) or a late response (latency greater than $1,000 \mathrm{msec}$ ) on fewer than $1 \%$ of the trials. The overall response accuracy was $83 \%$, and there were differences across conditions $[F(3,63)=7.35, p<.001]$. Fewer errors were made in the valid near condition $(15 \%$ error rate) that in the nonvalid near condition $[22 \%$ error rate; $t(21)=4.35, p<.01$ ], providing further support for a spatial cuing effect and again arguing against a speedaccuracy tradeoff. Slightly more errors were made in the object condition ( $16 \%$ error rate) than in the far condition ( $15.7 \%$ error rate), but the difference between them was not significant $[t(21)=0.3, p>.05]$.

\section{Discussion}

The present experiment replicated the important result from Experiment 2 that spatial and object-based attention 


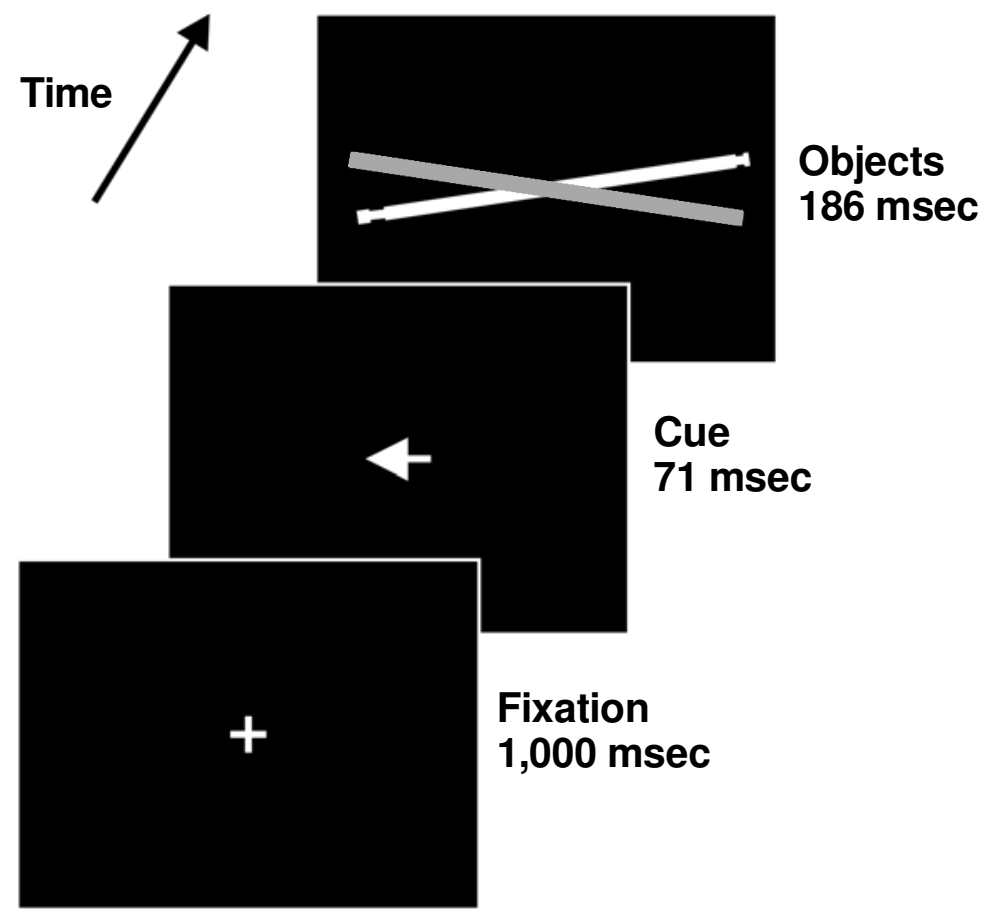

Figure 5. Sequence of events on a trial in Experiment 3. Here, attention was cued by a symbolic arrow cue that pointed to the side where the notches were most likely to appear. Otherwise, Experiment 3 was the same as Experiment 2.

can coexist. This finding was extended to include endogenously cued spatial attention. So, neither exogenously nor endogenously cued spatial attention eliminated objectbased attention. This lack of influence is at least suggestive that the mechanisms underlying object-based attention operate independently of the mechanisms serving both exogenous and endogenous attentional orienting.

\section{EXPERIMENT 4}

Experiments 2 and 3 showed that spatially cued attention and object-based attention could coexist when spatial attention was cued either exogenously or endogenously. But why is it that Lavie and Driver (1996) did not obtain this same finding? It was proposed earlier that the cue they used might have been an effective spatial cue and an effective object cue and, furthermore, might have cued both objects at the same time, which could have prevented detection of an object advantage. The results of Experiments 2 and 3 are consistent with this explanation, since the cues used there were unlikely to have been effective object cues and both a spatial cuing effect and an object advantage were found. However, there is still the possibility that the discrepancy between the results was not due to the differences between the cues used by Lavie and Driver and the ones used in the present study at all but, rather, to other aspects of their method, such as the proportion of object condition trials, the duration for which the objects were presented, and the objects themselves. These possibilities are considered next.
The discrimination stimuli (i.e., different colored dashes) in the experiments in which Lavie and Driver (1996) found an object advantage were on the same object for $25 \%$ of the trials. However, these discrimination stimuli were on the same object for only $10 \%$ of the trials in their cuing experiment. So, in the cuing experiment, where no object advantage was found, the condition proportions were more heavily weighted against object selection than in their other experiments, in which an object advantage was found. Also, in their cuing experiment, the object stimuli were presented for a shorter duration $(130 \mathrm{msec})$ than they were in their other experiments $(177 \mathrm{msec})$. It is possible that the objects could not exert much influence on selection if this shorter duration did not allow the subjects sufficient time to fully process the objects. So, not only is there no direct evidence that the method of their cuing experiment was capable of producing and detecting an object advantage without the cue, but also the method seems biased somewhat against it.

Since Lavie and Driver's (1996) explanation rests on a null effect and the expectation that their method could otherwise detect an object advantage, it is important to replicate their experiments while carefully controlling critical aspects of the method other than the presence of the cue. That is what was done in the present experiment. Experiment 2, Experiment 3, and the present experiment had identical condition proportions, and the objects were presented for the same durations. The important difference is that the present experiment used a cue modeled after the cue used by Lavie and Driver. Since Experiment 2 and Ex- 


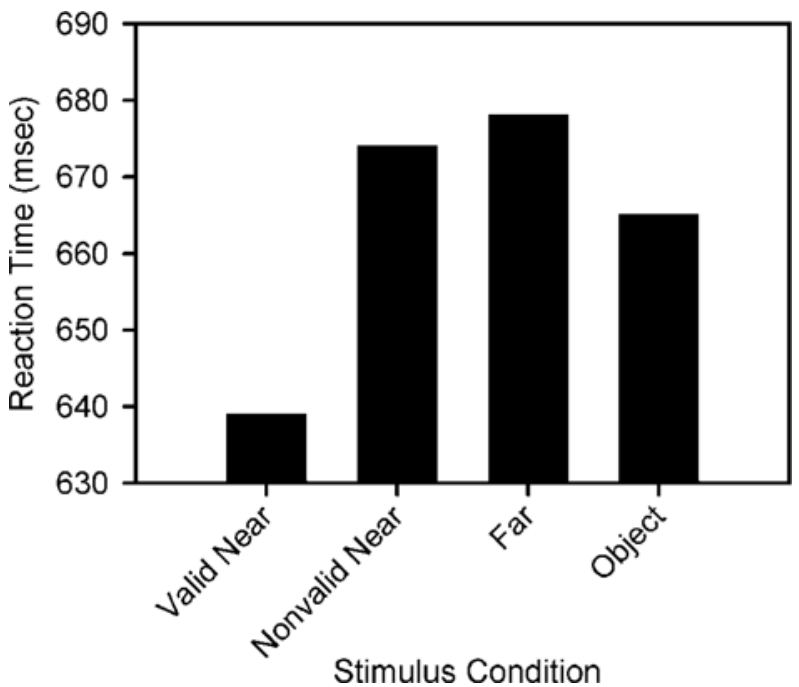

Figure 6. Results from Experiment 3. Spatial cuing in this experiment was accomplished using an endogenous central arrow. Both a cuing effect (difference between the two leftmost bars) and an object advantage (difference between the two rightmost bars) were observed.

periment 3 each produced an object advantage, any modulation of that object advantage here can be attributed only to the difference between the types of cues. If an object advantage is found, it would mean that the type of cue Lavie and Driver used is probably not the reason the ob- ject effect was eliminated in their experiment. Instead, it becomes more likely that the condition proportions, the object duration, or the objects they used were responsible for preventing them from producing and detecting an object advantage (these issues are pursued in Experiment 5).

\section{Method}

Subjects. In return for class credit, 16 undergraduates participated in a single 1-h session. They all had normal or corrected-tonormal vision, were naive as to the purposes of the experiment, and did not participate in any prior experiment in this study.

Procedure. This experiment used the bar objects oriented as in Experiment 1 but was otherwise identical to Experiments 2 and 3, with the exception of the cue. As is shown in Figure 7, the bars were cued by presenting the two ends (i.e., the extreme $0.4^{\circ}$ ) of the bars on the cued side of the display $71 \mathrm{msec}$ prior to presenting the rest of the display.

\section{Results}

Mean reaction times for the valid near, nonvalid near, far, and object conditions are shown in Figure 8. The subjects were quickest to judge notch size in the valid near condition $(655 \mathrm{msec})$, next quickest in the nonvalid near condition $(670 \mathrm{msec})$, next quickest in the object condition $(672 \mathrm{msec})$, and slowest in the far condition [684 msec; $F(3,45)=13.2, p<.001]$. Comparing the far condition with the object condition shows that the object advantage was $12 \mathrm{msec}$ : The subjects were quicker to make their judgment in the object condition, in which the notches were both on the same bar, than in the far condition, in which the notches were on different bars but at a distance

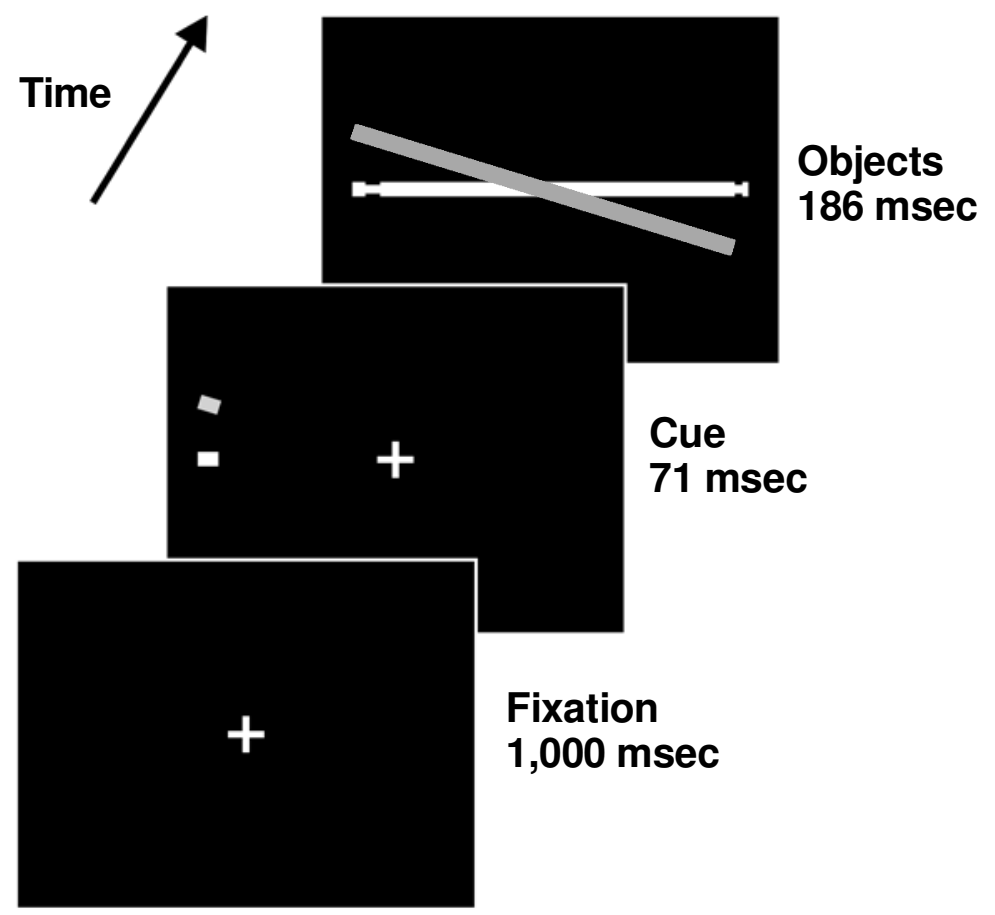

Figure 7. Sequence of events on a trial in Experiment 4. Here, attention was cued by presenting the ends of the bar objects on the side where the notches were most likely to appear. This cue was modeled after the one used by Lavie and Driver (1996). Otherwise, Experiment 4 was the same as Experiments 2 and 3. 
from each other similar to that in the object condition $[t(15)=3.591, p<.005]$. There was also a spatial cuing effect of 14 msec shown by the comparison of the valid near and nonvalid near conditions $[t(15)=5.123$, $p<.001]$. There was no difference between the nonvalid near and the object conditions $[t(15)=0.493, p>.05]$, perhaps in part owing to masking from the cue (see the Results section from Experiment 1 for a discussion of this possibility).

The subjects made a premature response (latency less than $100 \mathrm{msec}$ ) or a late response (latency greater than $1,000 \mathrm{msec}$ ) on fewer than $1 \%$ of the trials. The overall response accuracy was $85 \%$, and errors differed across condition $[F(3,45)=9.59, p<.001]$. Fewer errors were made in the valid near condition ( $14 \%$ error rate) than in the nonvalid near condition $[23 \%$ error rate; $t(15)=4.0$, $p<.01$ ], providing further evidence of a spatial cuing effect. There also were slightly fewer errors made in the object condition ( $13 \%$ error rate) than in the far condition ( $14 \%$ error rate), but not reliably so $[t(15)=0.9, p>.05]$.

\section{Discussion}

In the present experiment, both a spatial cuing effect and an object effect were found, using a type of cue similar to that used by Lavie and Driver (1996), in which a portion of each object is shown prior to the appearance of the complete objects. This is a third result showing that spatial attention and object-based attention can coexist. The present findings are thus inconsistent with Lavie and Driver's finding that focused spatial attention eliminates objectbased attention. Because an object advantage was found in the present experiment with a cuing technique similar to Lavie and Driver's, it seems that the differences between the two findings are not due to differences in the type of cue used to summon attention. This leaves open the possibility that the differences were due to condition proba-

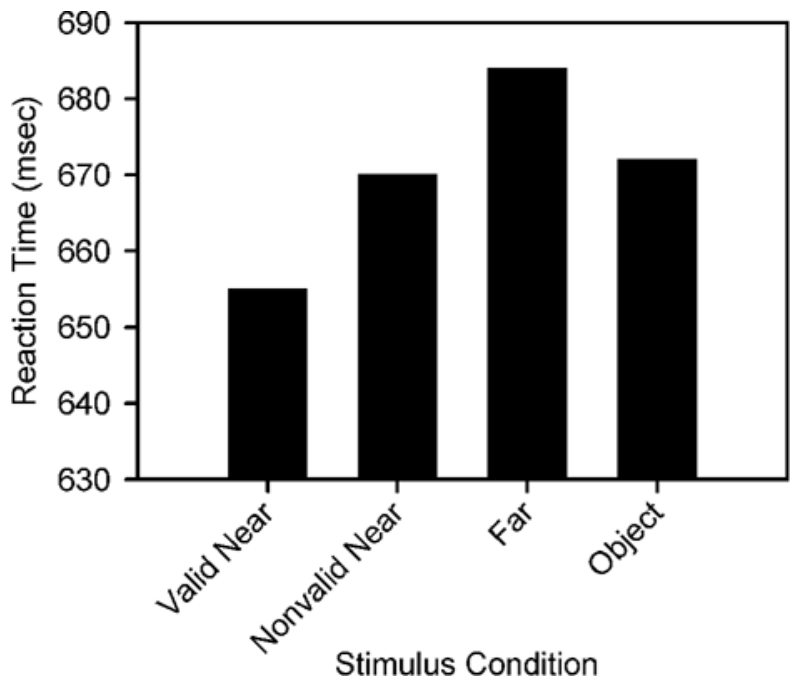

Figure 8. Results from Experiment 4 showing both a spatial cuing effect and an object advantage, as in Experiments 2 and 3. bilities, object display duration, stimulus qualities, or some combination of these factors. In the next experiment, we examined object display duration.

\section{EXPERIMENT 5}

As was noted earlier, Lavie and Driver (1996) presented object stimuli for $130 \mathrm{msec}$ in their experiment in which cuing was used and in which no object advantage was observed; however, they presented the stimuli for $177 \mathrm{msec}$ in their experiments without cuing but in which an object advantage was obtained. This leaves open the possibility that the difference in stimulus durations is responsible for the absence (or presence) of an object advantage. In particular, it might be that the shorter exposure duration used by Lavie and Driver in their experiment with cuing did not allow enough time for the segmentation of the scene into objects and, hence, only a cuing benefit was observed. To examine that possibility, we repeated Experiment 4 with only one difference: Here, we presented the bar stimuli for $129 \mathrm{msec}$, as compared with $186 \mathrm{msec}$ in Experiment 4.

\section{Method}

Subjects. In return for class credit, 16 undergraduates participated in a single 1-h session. They all had normal or corrected-tonormal vision, were naive as to the purposes of the experiment, and had not participated in any prior experiment in this study.

Procedure. All aspects of this experiment were identical to those in Experiment 4, except that, here, the bar objects were presented for $129 \mathrm{msec}$.

\section{Results}

Mean reaction times for the valid near, nonvalid near, far, and object conditions are shown in Figure 9. Overall, there was a main effect of condition $[F(3,45)=10.4, p<$ $.001]$. There was a $27-\mathrm{msec}$ cuing effect, with valid near faster than nonvalid near $[t(15)=4.1, p<.005]$. There was a nonsignificant 4.6-msec object cost [based on a comparison of the object and the far conditions; $t(15)=0.76$, n.s.]. Overall the error rate was $16 \%$, with some differences across conditions $[F(3,45)=6.46, p<.005]$. Fewer errors were made in the valid near condition $(12 \%)$ than in nonvalid near condition [20\%; $t(15)=3.1, p<.01]$. Error rates in the far $(16 \%)$ and object $(15 \%)$ conditions did not differ from one another $[t(15)=1.3$, n.s. $]$.

\section{Discussion}

In the present experiment, unlike all of the earlier experiments reported here, we failed to obtain an object benefit. That is, the subjects were not quicker to compare two elements on one object relative to one element on each of two objects. Importantly, the absence of an object benefit occurred here at the same time that a cuing benefit was observed. Thus, in the present experiment, we succeeded in replicating the pattern of results reported by Lavie and Driver (1996). The present experiment was identical to Experiment 4, except that the duration of the bar objects was shorter here (129 msec, as compared with $186 \mathrm{msec}$ ). Because that was the only difference between the experi- 


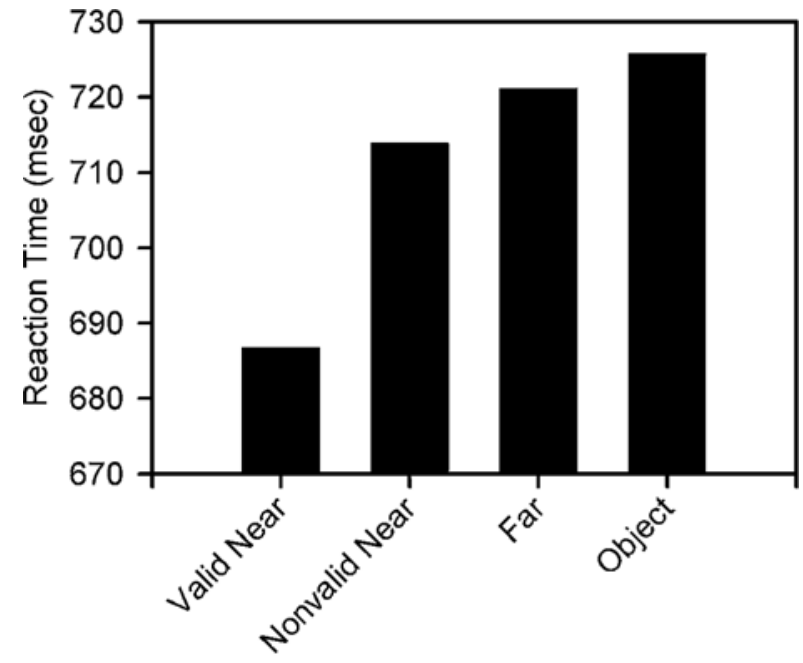

Stimulus Condition

Figure 9. Results from Experiment 5. A spatial cuing effect is revealed by comparing the valid near and the nonvalid near conditions, but no object advantage could be seen by comparing the far and the object conditions.

ments, we believe that the shorter duration either did not permit establishment of object representations or did not allow enough time for object representations to be established prior to the availability of the information needed to perform the task through some alternate representation. Importantly, the shorter duration was the same as that used by Lavie and Driver in their experiment in which no object benefit was found, consistent with the conclusion that their result was caused by the shorter duration used there, as compared with their other experiments.

\section{GENERAL DISCUSSION}

\section{Summary of Findings}

The present study shows that spatially directed attention and object-based attention can exist simultaneously: Object-based selection was observed in the absence of focused spatial attention, as well is in the presence of focused attention as directed by three different types of cues. Experiment 1 showed that the object stimuli used throughout the study could effectively produce an object advantage (as revealed by faster comparison of two features when they were on a single object than when they were on two separate objects). In Experiment 2, spatial attention was directed to one side of the display by briefly presenting three dots that appeared near the region that would soon be occupied by the notched bar objects. The subjects were faster and more accurate to judge the relative size of the notches when both notches were presented on the cued side of the display than when they were both presented on the uncued side (demonstrating focused spatial attention as summoned by the cue). Importantly, the subjects were faster to compare notch size when the notches were both on the same bar object than when they were a similar distance apart but on different objects, demonstrating an object-based effect. So, both spatially directed attention and object-based attention were able to operate simultaneously, with the object advantage extending beyond the area that was cued. Experiment 3 was similar to Experiment 2 but employed an endogenous central arrow cue to direct attention to one part of the display. And in Experiment 4 , attention was cued by presenting one end of each bar in advance, similar to the method used by Lavie and Driver (1996). In each of those experiments, a spatial cuing effect and an object advantage were observed. Finally, in Experiment 5, the stimulus duration was shortened to match that used by Lavie and Driver in their experiment in which no object advantage was found, and indeed, the reduced duration caused the object effect to disappear. We conclude that with the shorter duration, the information needed to perform the discrimination task became available prior to completion of segmentation of the scene into objects. As a result, there was no object effect in Experiment 5.

In Experiments 2-4 (with spatial cuing), the object advantage was 11,12 , and $13 \mathrm{msec}$, which did not differ from the 15-msec object advantage observed with no cuing in Experiment $1[F(3,69)<1]$. Thus, the focusing of attention did not reduce the object advantage measurably.

\section{Relation to Previous Findings}

The present results help to resolve a controversy in the literature on selective attention. On the one hand, results such as those reported by Lavie and Driver (1996) suggest that the effects of objects on selection might be eliminated by the active focusing of attention. Those results are consistent, in a general way, with findings that show the existence of attentional control settings that might permit a person to enable or disable sensitivity to certain types of stimulus information (e.g., Folk et al., 1992). On the other hand, Lavie and Driver's results seem inconsistent with results such as those reported by Egly et al. (1994; see also Abrams \& Law, 2000), in which objects exerted an influence even while attention was focused on part of the display. Consistent with the latter results are the findings of Lamy (2000), who failed to replicate Lavie and Driver's results when using a nearly identical experimental method.

Taken together, our results support an important role of objects in the guidance of selective attention. The perceived objects in a scene determine in part the locations to which attention will be allocated, even while attention is specifically directed to a part of the scene containing only a portion of the objects that are visible. At the same time, we may also have identified a boundary condition that may affect the use of an object-based representation: When the stimulus duration is very brief, attention may be guided by a representation that is not object based.

\section{Endogenous and Exogenous Cuing}

The present results also bear on the distinction between stimulus-driven selection as produced by exogenous cues and goal-directed selection as driven by endogenous cuing. Despite the many differences between them (see Abrams \& Law, 2000, for a discussion), there is currently some de- 
bate regarding the extent to which the two types of orienting may activate similar attentional mechanisms. In particular, Macquistan (1997) failed to find an object advantage when using endogenous cues in a paradigm similar to that used by Egly et al. (1994; Egly et al. had used exogenous cues). However, Abrams and Law showed, in several experiments, that endogenous attentional cues can indeed activate the attentional system in such a way as to permit an object advantage. The present results provide further support for the coexistence of goal-directed attentional selection and object-based orienting: In Experiment 3, with endogenous cues, the object advantage was $13 \mathrm{msec}-$ comparable to the 11- and 12-msec object advantages that had been observed in Experiments 2 and 4 with exogenous cues. Thus, although there are clearly differences between stimulus-driven and goal-directed orienting mechanisms, they do not appear to differ with respect to the presence of object-based effects.

\section{Implications for Models of Attention}

The present results have important implications for our understanding of the mechanisms underlying selective attention. In particular, if object-based selection did not occur outside the focus of attention (as had been reported by Lavie \& Driver, 1996), it might be necessary to propose that spatial selection operates prior to the point in processing at which objects can exert their effects. Such a proposal might be needed to account for the supposed absence of an effect of objects outside the focus of spatial attention. However, that proposal would be inconsistent with some extant results that suggest that objects exert their effects very early in the visual processing stream (e.g., Abrams \& Law, 2000; Davis \& Driver, 1994). However, our results are entirely consistent with the view that object effects occur early in processing, because we found that they can continue to affect attention beyond the selected region for several different types of cues. Given that we live in a world in which parts of objects usually do not exist in isolation from the objects themselves, it is perhaps not surprising that objects can exert such a strong effect on our attentional selection mechanisms.

\section{REFERENCES}

Abrams, R. A., \& Dobkin, R. S. (1994). Inhibition of return: Effects of attentional cuing on eye movement latencies. Journal of Experimental Psychology: Human Perception \& Performance, 20, 467-477.

Abrams, R. A., \& Law, M. B. (2000). Object-based visual attention with endogenous orienting. Perception \& Psychophysics, 62, 818-833.

Abrams, R. A., \& Pratt, J. (2000). Oculocentric coding of inhibited eye movements to recently attended locations. Journal of Experimental Psychology: Human Perception \& Performance, 26, 766-788.

BAYlis, G. C., \& Driver, J. (1993). Visual attention and objects: Evidence for hierarchical coding of locations. Journal of Experimental Psychology: Human Perception \& Performance, 19, 451-470.

Breitmeyer, B. G., \& GANZ, L. (1976). Implications of sustained and transient channels for theories of visual pattern masking, saccadic suppression, and information processing. Psychological Review, 83, $1-36$.

Christ, S. E., McCrae, C. S., \& Abrams, R. A. (2002). Inhibition of return in static and dynamic displays. Psychonomic Bulletin \& Review, 9, $80-85$.
Dagenbach, D., Goolsby, B., Neely, C. A., \& Dudziak, K. M. (1997, November). Further studies of attention to space and objects with endogenous cuing. Poster presented at the 38th Annual Meeting of the Psychonomic Society, Philadelphia.

DAvis, G., \& DrIVER, J. (1994). Parallel detection of Kanizsa subjective figures in the human visual system. Nature, 371, 791-793.

DunCAN, J. (1984). Selective attention and the organization of visual information. Journal of Experimental Psychology: General, 113, 501517.

Egly, R, Driver, J., \& Rafal, R. D. (1994). Shifting visual attention between objects and locations: Evidence from normal and parietal lesion subjects. Journal of Experimental Psychology: General, 123, 161-177.

Folk, C. L., \& Remington, R. (1999). Can new objects override attentional control settings? Perception \& Psychophysics, 61, 727-739.

Folk, C. L., Remington, R. W., \& Johnston, J. C. (1992). Involuntary covert orienting is contingent on attentional control settings. Journal of Experimental Psychology: Human Perception \& Performance, 18, 1030-1044.

Gellatly, A., Cole, G., \& Blurton, A. (1999). Do equiluminant object onsets capture visual attention? Journal of Experimental Psychology: Human Perception \& Performance, 25, 1609-1624.

JonIDES, J. (1981). Voluntary vs. automatic control over the mind's eye's movement. In J. [B.] Long \& A. [D.] Baddeley (Eds.), Attention and performance IX (pp. 187-203). Hillsdale, NJ: Erlbaum.

Kahneman, D., Treisman, A., \& GibBs, B. (1992). The reviewing of object files: Object-specific integration of information. Cognitive Psychology, 24, 175-219.

LAMY, D. (2000). Object-based selection under focused attention: A failure to replicate. Perception \& Psychophysics, 62, 1272-1279.

LAVIE, N., \& DRIVER, J. (1996). On the spatial extent of attention in objectbased visual selection. Perception \& Psychophysics, 58, 1238-1251.

Macquistan, A. D. (1997). Object-based allocation of visual attention in response to exogenous, but not endogenous, spatial precues. Psychonomic Bulletin \& Review, 4, 512-515.

Muller, H. J., \& RabbitT, P. M. A. (1989). Reflexive and voluntary orienting of visual attention: Time course of activation and resistance to interruption. Journal of Experimental Psychology: Human Perception \& Performance, 15, 315-330.

Oonk, H. M., \& ABrams, R. A. (1998). New perceptual objects that capture attention produce inhibition of return. Psychonomic Bulletin \& Review, 5, 510-515.

Posner, M. I. (1980). Orienting of attention. Quarterly Journal of Experimental Psychology, 32, 3-25.

Posner, M. I., \& Cohen, Y. (1984). Components of visual orienting. In H. Bouma \& D. G. Bowhuis (Eds.), Attention and performance $X$ : Control of language processes (pp. 531-556), Hillsdale, NJ: Erlbaum.

Rafal, R. D., Calabresi, P. A., Brennan, C. W., \& Sciolto, T. K. (1989). Saccade preparation inhibits reorienting to recently attended locations. Journal of Experimental Psychology: Human Perception \& Performance, 15, 673-685.

Riggio, L., \& KIRSNER, K. (1997). The relationship between central cues and peripheral cues in covert visual orienting. Perception \& Psychophysics, 59, 885-899.

Tipper, S. P., Driver, J., \& Weaver, B. (1991). Object-centered inhibition of return of visual attention. Quarterly Journal of Experimental Psychology, 43A, 289-298.

VeCERA, S. P. (1994). Grouped locations and object-based attention: Comment on Egly, Driver, and Rafal (1994). Journal of Experimental Psychology: General, 123, 316-320.

Yantis, S., \& Hillst rom, A. P. (1994). Stimulus-driven attentional capture: Evidence from equiluminant visual objects. Journal of Experimental Psychology: Human Perception \& Performance, 20, 95-107.

YANTIS, S., \& JoNIDES, J. (1990). Abrupt visual onsets and selective attention: Voluntary vs. automatic allocation. Journal of Experimental Psychology: Human Perception \& Performance, 16, 121-134.

(Manuscript received March 22, 2000; revision accepted for publication January 30, 2002.) 